\title{
Polymers in Drug Delivery
}

\author{
Apurva Srivastava, Tejaswita Yadav, Soumya Sharma, Anjali Nayak, Akanksha Kumari, \\ Nidhi Mishra* \\ Department of Applied Science, Indian Institute of Information Technology, Deoghat, Jhalwa, Allahabad, India \\ Email: *nidhimishra@iiita.ac.in
}

Received 7 November 2015; accepted 11 January 2016; published 14 January 2016

Copyright (C) 2016 by authors and Scientific Research Publishing Inc.

This work is licensed under the Creative Commons Attribution International License (CC BY). http://creativecommons.org/licenses/by/4.0/

c) (i) Open Access

\begin{abstract}
Polymers are being used extensively in drug delivery due to their surface and bulk properties. They are being used in drug formulations and in drug delivery devices. These drug delivery devices may be in the form of implants for controlled drug delivery. Polymers used in colloidal drug carrier systems, consisting of small particles, show great advantage in drug delivery systems because of optimized drug loading and releasing property. Polymeric nano particulate systems are available in wide variety and have established chemistry. Non toxic, biodegradable and biocompatible polymers are available. Some nano particulate polymeric systems possess ability to cross blood brain barrier. They offer protection against chemical degradation. Smart polymers are responsive to atmospheric stimulus like change in temperature; pressure, $\mathrm{pH}$ etc. thus are extremely beneficial for targeted drug delivery. Some polymeric systems conjugated with antibodies/specific biomarkers help in detecting molecular targets specifically in cancers. Surface coating with thiolated PEG, Silica-PEG improves water solubility and photo stability. Surface modification of drug carriers e.g. attachment with PEG or dextran to the lipid bilayer increases their blood circulation time. Polymer drug conjugates such as Zoladex, Lupron Depot, On Caspar PEG intron are used in treatment of prostate cancer and lymphoblastic leukemia. Polymeric Drug Delivery systems are being utilized for controlled drug delivery assuring patient compliance.
\end{abstract}

\section{Keywords}

Polymeric Drug Delivery, Biocompatible Polymers, Smart Polymers, Polymeric Implants, Polymeric Drug Formulations

\section{Introduction}

Polymers have become an integral part of drug delivery systems due to their improved pharmacokinetic properties. They have better circulation time than conventional small drug molecules thus target tissue more specifi-

*Corresponding author. 
cally. Tremendous use of polymers has been witnessed in the area of polymer therapeutics and Nano medicines [1]. Polymers in reservoir based drug delivery systems have shown immense progress in the form of hydrogels and liposomes. Diffusion based drug delivery systems (as shown in Figure 1) and solvent activated drug delivery systems are the other areas being explored for utilizing the polymers [1]. In diffusion based drug delivery systems drug is dissolved in a non-swell able system or a fully swollen matrix which does not decompose during their activation time. Solvent activated systems like hydrogels swell and release the drug when exposed to aqueous environment; this mechanism is depicted in Figure 2. They are hydrophilic in nature [2]. Biocompatible polymers offer a safe passage for drug delivery due to their well engineered molecular architecture according to the transitions in the underlying mechanisms of the biological process. Biodegradable polymers break due to cleavage of covalent bonds between them and bio erodible polymers bring about erosion of the polymer due to dissolution of linking chains without bringing about any change in chemical structure of the molecule [2]. Various drug release mechanisms can be studied comparatively in Figure 3. Polymers serving as drug carrier should be water soluble, nontoxic and non-immunogenic. They work passively in minimizing drug degradation and improving circulation time. Another important issue is the safe excretion of the drug [1]. If the polymer is nondegradable it should be ensured that it is not accumulated within the body and if it is degradable the broken components should be such that they lie below renal threshold level, non toxic and should not produce any immune response [1]. Polymers mimicking biological systems respond to external stimulus such change in $\mathrm{pH}$ or temperature and as a result their properties such as solubility, hydrophobic/ hydrophilic balance, release of biomolecule (drug molecule) and conformation are altered [1].

\section{Various Polymers Used in Drug Delivery}

\section{PLGA}

In past two decades poly lactic-co-glycolic acid (PLGA) has been among the most attractive polymeric candidates used to fabricate devices for drug delivery and tissue engineering applications. PLGA is biocompatible and

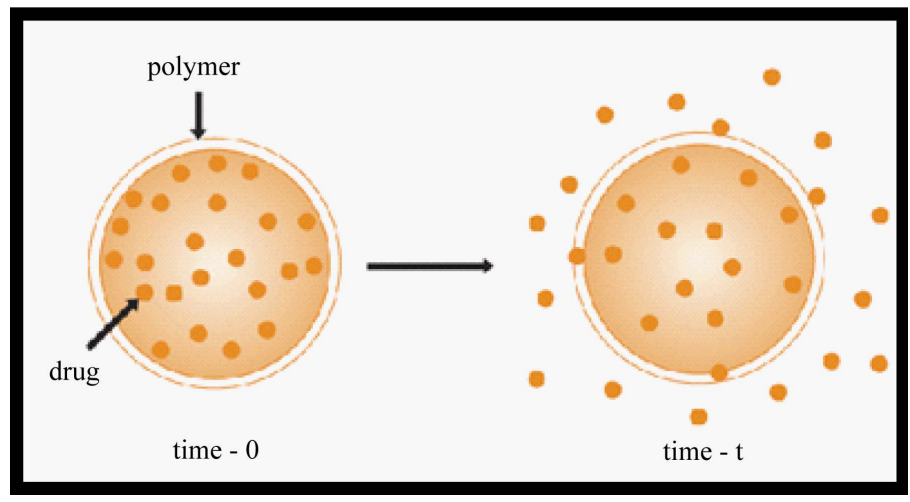

Figure 1. Diffusion based drug delivery system [6].

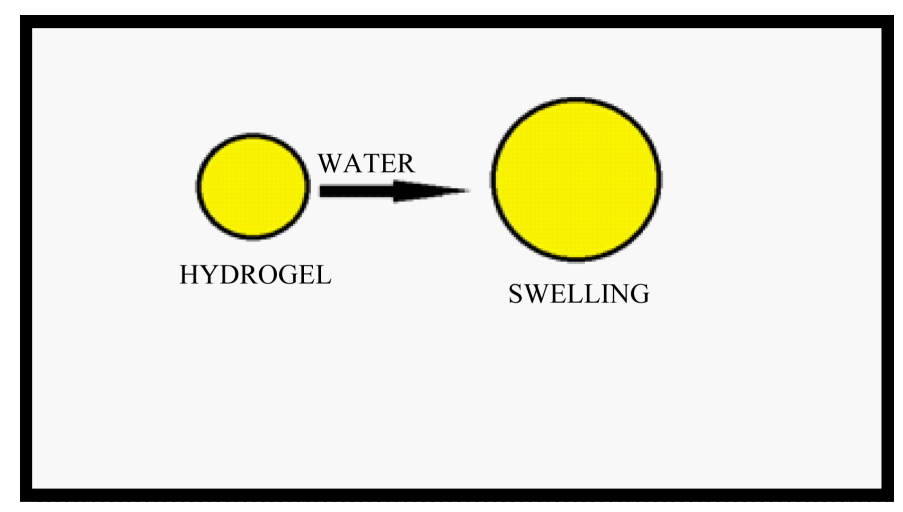

Figure 2. Hydrogel based drug delivery system. 


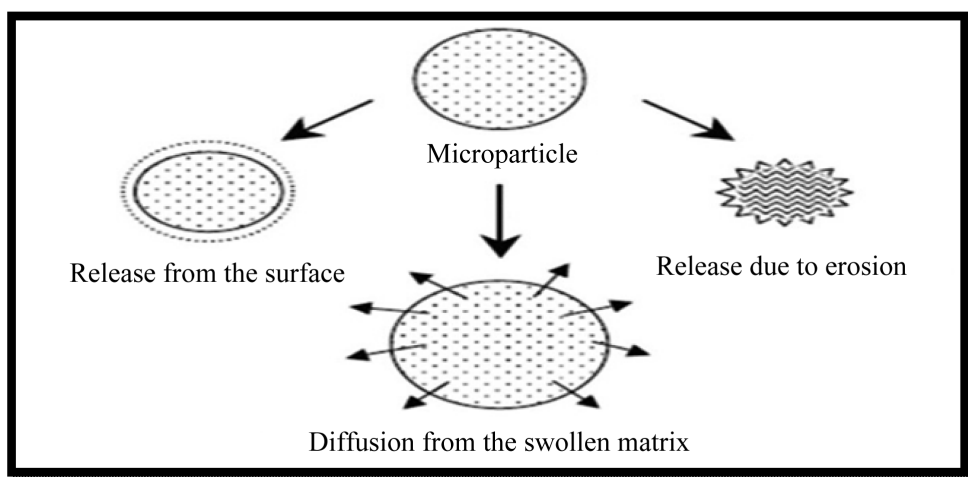

Figure 3. Various drug release mechanisms [5].

biodegradable, exhibits a wide range of erosion times, has tunable mechanical properties.

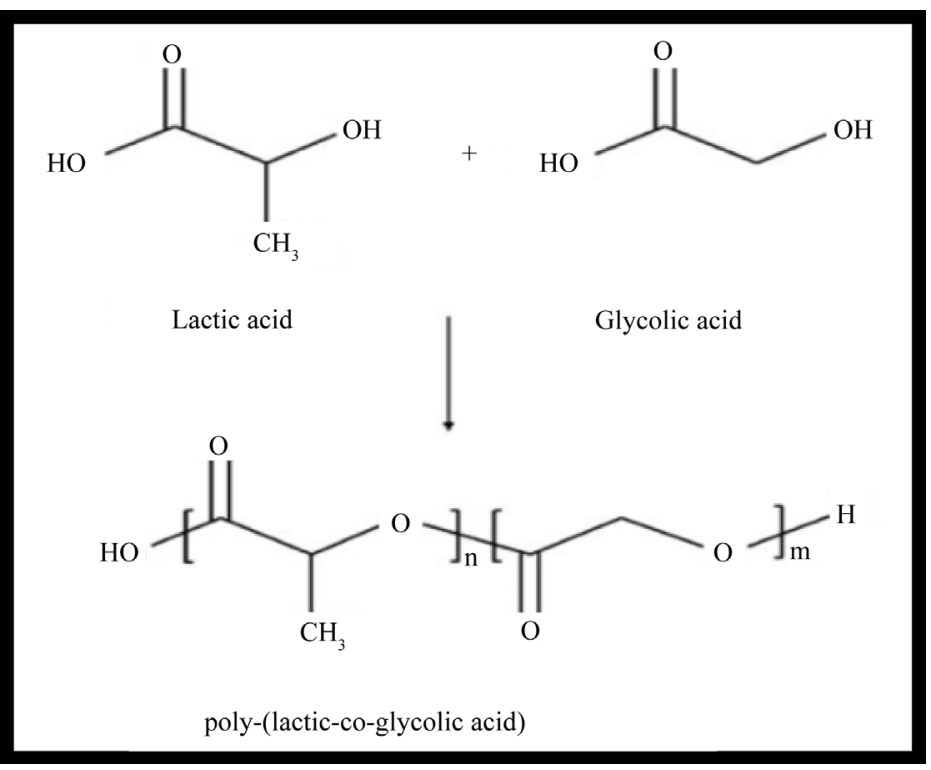

\section{PGA(poly glycolic acid)}

Polyglycolic acid (PGA) is a biodegradable, thermoplastic polymer and the simplest linear, aliphatic polyester. It can be prepared starting from glycolic acid by means of polycondensation or ring-opening polymerization. PGA has been known since 1954 as a tough fiber-forming polymer.

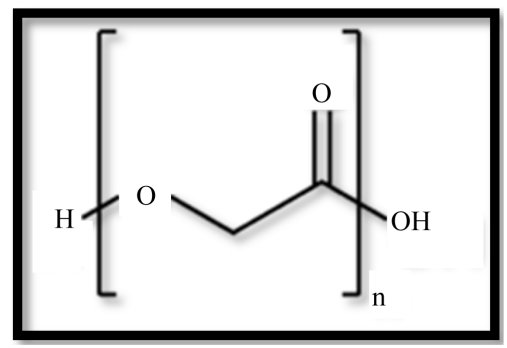

PGA(poly glycolic acid)

\section{Poly-l-glutamic acid}

Polyglutamic acid (PGA) is a polymer of the amino acid glutamic acid (GA). Gamma PGA is formed by bacterial fermentation. 
Gamma PGA has a wide number of potential uses ranging from food and medicine to water treatment. It is widely being used as a drug delivery system in cancer treatment.

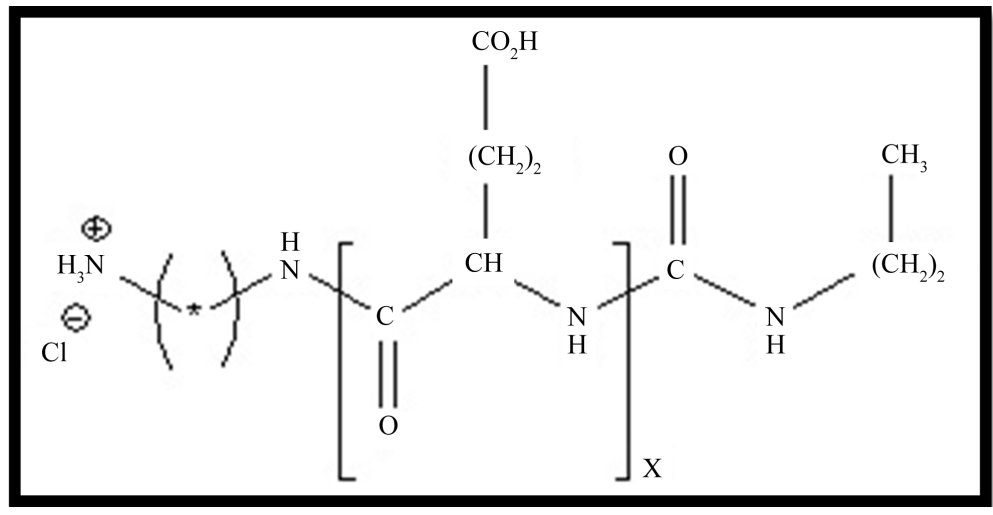

Poly-l-glutamic acid

\section{Polylactic acid}

It is a biodegradable thermoplastic aliphatic polyester derived from renewable resources, such as co $\mathrm{r} n$ starch (in the United States and Canada), tapioca roots, chips or starch (mostly in Asia), or sugarcane (in the rest of the world).

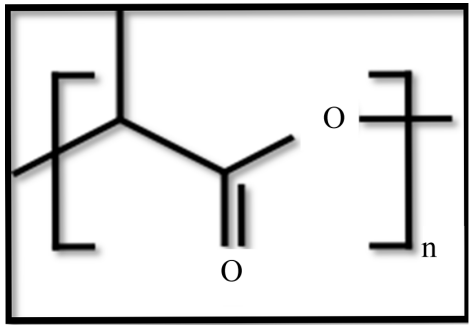

Poly lactic acid

PNIPAAm [Poly $(N$-isopropylacrylamide $)]$

It is a temperature responsive polymer that was first synthesized in the 1950s.It can be synthesized from $\mathrm{N}$-isopropylacrylamide which is commercially available. It is synthesized via free radical polymerization and is readily functionalized making it useful in a variety of applications.

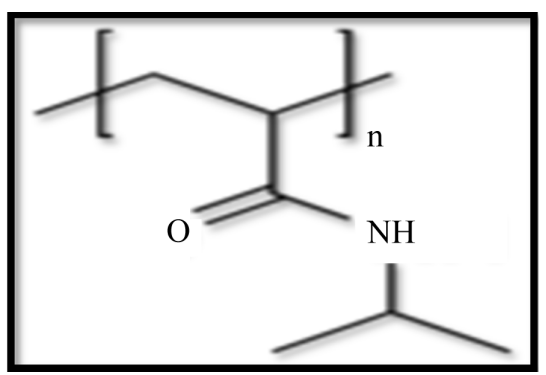

PNIPAAm [Poly( $N$-isopropylacrylamide)]

pHEMA[Poly 2-hydroxyethyl methacrylate]

It is a polymer that forms a hydrogel in water. Poly (hydroxyethyl methacrylate). It was invented by Drahoslav Lim and Otto Wichterle for biological use. Together they succeeded in preparing a cross-linking gel which absorbed up to $40 \%$ of water, exhibited suitable mechanical properties and was transparent. They patented this 
material in 1953.

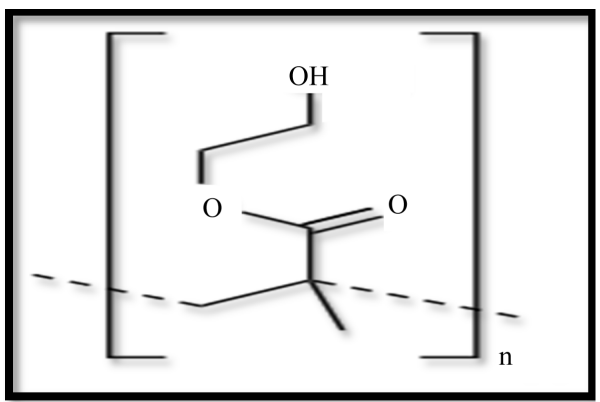

pHEMA[Poly 2-hydroxyethyl methacrylate]

\section{PPy [Polypyrrole]}

It is a type of organic polymer formed by polymerization of pyrrole. Polypyrroles are conductin polymers, related members being polythiophene, polyaniline, and polyacetylene. The Nobel Prize in Chemistry was awarded in 2000 for work on conductive polymers including polypyrrole. The first examples of polypyrroles were reported in 1963 by Weiss and coworkers.

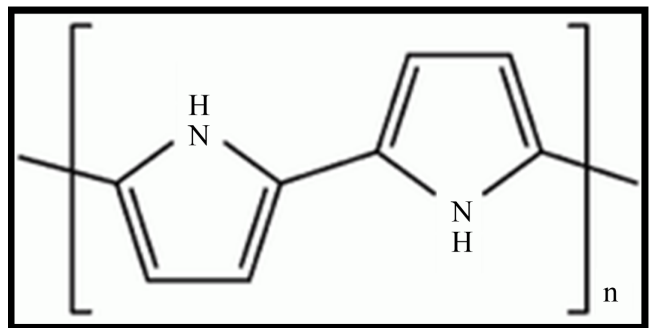

PPy [Polypyrrole]

\section{PAMAM [Poly (amidoamine)]}

It is a class of dendrimer which is made of repetitively branched subunits of amide and amine functionality. PAMAM dendrimers, sometimes referred to by the trade name Starburst, have been extensively studied since their synthesis in 1985, and represent the most well-characterized dendrimer family as well as the first to be commercialized. Like other dendrimers, PAMAMs have a sphere-like shape overall, and are typified by an internal molecular architecture consisting of tree-like branching, with each outward "layer", or generation, containing exponentially more branching points

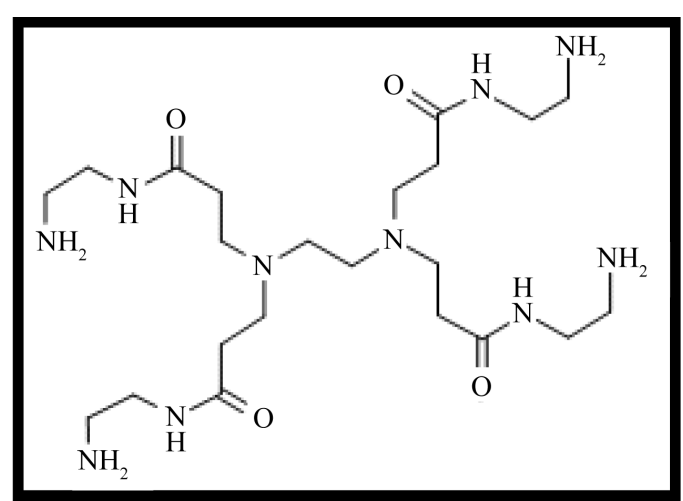

PAMAM [Poly (amidoamine)]

\section{DEXTRAN}




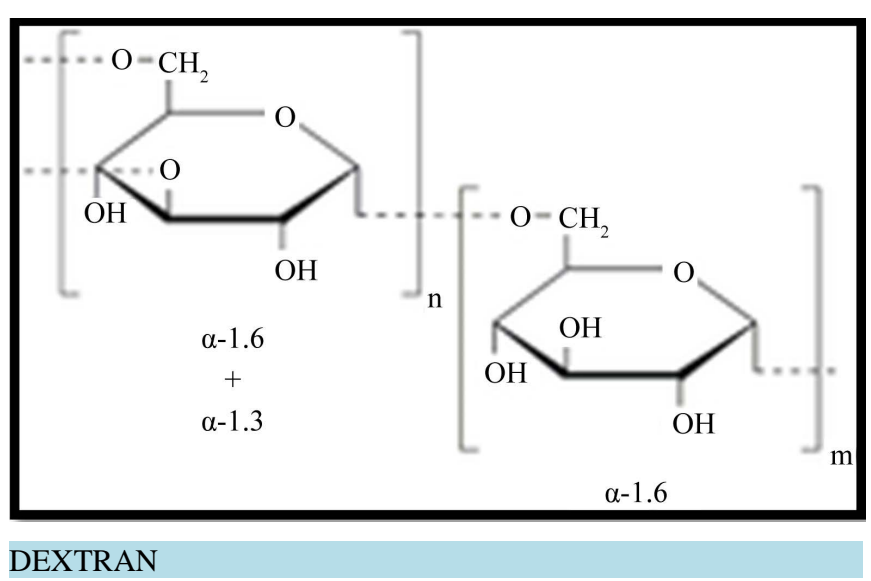

STRUCTURE: Dextran is can be defined by Leuconostoc mesenteroides (lactic-acid bacteria with the help of which dextran is synthesized using sucrose) which contains a glucan which is (16)-linked and has side chains that are attached to the backbone of 3-positions of glucose units. The straight chain consists of $\alpha-1,6$ glycosidiclinkages between the molecules of glucose. The branching starts from $\alpha-1,3$ linkages [1].

DISCOVERY HISTORY - Allene Jeanes was a scientist at the USA's Northern Regional Research Lab in 1940. One day a company of soft drinks sent them one sample of their product which became thick and sticky. It was found out that this stickiness was due to the presence of a bacterium that converted the sugar present in the soda to dextran. This bacteria was assumed to have come from the dental plaque of one of the workers at the factory. The scientist then discovered that bacterium can be grown in the lab in a sugar solution which will make lots of dextran. This was then purified, dried and sent to korea which helped the soldiers to survive and helped them to heal.

\section{Conventional Use of Polymers in Drug Delivery}

Conventional drug delivery systems use doses of drugs in form of capsules, tablets which are formed by compression, coating and encapsulation of bioactive drug molecules [2]. Polymers play a versatile role in such conventional formulations; they serve as binding agents in capsules, film coating agents in tablets and viscosity enhancers in emulsions and suspensions. Some of the polymers given along with bioactive drug molecules include cellulose derivatives, poly (N-vinyl pyrrolidone) and poly (ethylene glycol) PEG.

\section{Smart Polymers}

Smart polymers are those which exhibit change depending upon the change in environmental conditions. In medicine stimuli responsive polymers show the change in their properties in response to the change in biological conditions [7]. The various stimuli may be temperature, pressure, $\mathrm{pH}$, electric field, magnetic field, light, change in concentration, ionic strength, redox potential etc. [1]. Responses to such stimuli include dissolution, precipitation, swelling, change in conformation, and change in hydrophobic and hydrophilic properties. There is change in $\mathrm{pH}$ along the GI tract which is considered during design of oral drug delivery systems [8]. The cancerous tissue and swelled tissue show a drastic variation in $\mathrm{pH}$. Polymer bound drugs are released in such tissues due to deprotonation/protonation of complex polymer structure under the conditions of altered $\mathrm{pH}$. Poly (methacrylic acid) jointure with PEG, referred to as P (MAA-g-EG) has been used for oral protein delivery [3]. Similarly temperature responsive polymers bring about change in hydrophilicity/hydrophobicity of polymers enhancing their membrane permeation. PNIPAAm, is a thermoresponsive polymer and it is being thoroughly studied for its ability to undergo a negative temperature-dependent phase transition. Below its lower critical solution temperature, PNIPAAm exists as a hydrophilic coil, whereas above the LCST, PNIPAAm chains convert sharply into a hydrophobic globule [3]. This volume phase transition arises from the hydrophilic/hydrophobic balance of polymer chains, which is modulated by formation and disruption of electrostatic and hydrophobic interactions both within and among the molecules. Alteration in polymer properties can be used to:

- Adhere to the cell surface 
- Break down cellular membrane and

- Release biologically active compound.

Stimuli responsive polymers can be broadly categorized into micelles, polyplexes and polymer drug conjugates.

\section{Polymers in Novel Drug Delivery Systems}

Chemical engineers, pharmacologists and scientists are using polymers for developing controlled drug release systems and sustained release formulations [3]. Novel drug delivery systems include micelles, dendrimers, liposomes, polymeric nanoparticles, cell ghosts, microcapsules and lipoproteins. Recent advancements in polymer based encapsulations and controlled drug release systems help in regulating drug administration by preventing under or overdosing [2]. These advanced systems play a promising role in improving bioavailability, minimizing side effects and other types of inconveniences caused to the patients. Studies need to be performed in the areas of surface and bulk properties of polymers as these properties govern their utilization various applications. Role of polymers in drug delivery will grow steeply in future to handle various unsolved issues. These issues may include site specific drug delivery in subcellular organelles, harnessing chemical, physical and biological properties efficiently to optimize drug administrations. Nano composites have shown to penetrate deep blood brain barriers [2].

Through this paper we emphasize on the role of polymers in existing and novel drug delivery systems both as formulations and in devices, their advantages and limitations.

\section{Advantages:}

1. Polymers used in colloidal drug carrier systems, consisting of small particles, show great advantage in drug delivery systems because of optimized drug loading and releasing property [1].

2. A polymer (natural or synthetic) is aggregated with a drug in controlled drug delivery and hence it gives a effective and controlled dose of dug avoiding overdose [1].

3. The degradable polymers are ruptured into biologically suitable molecules that are assimilated and discarded from the body through normal route.

4. Reservoir based polymers is advantageous in various ways like it increase the solubility of incompetently soluble drugs and it lowers the antagonistic side effects of drugs [2].

5. Magneto-optical polymer coated and targeted nanoparticles are multimodal (optical and MRI detection) while Quantum Dots are only optically detectable.

6. Some Quantum dots contain Cd which is known to be toxic to humans. Magneto/optical nanoparticles whether polymer coated or targeted are composed of iron oxides/polymers which are known to be safe, therefore have great future.

7. Dextrans is the common polymer used for coating of iron oxide (plasma expander and affinity for iron) and are used for treatment of iron anaemias since 1960 and is still in operation.

8. In controlled release, some of the polymers like polyurethanes for elasticity, polysiloxanes for insulating ability are used for their intended non-biological physical properties.

9. Current polymers like Poly 2-hydroxy ethyl methacrylate, polyvinyl alcohol, Polyethylene glycol are used because of their inert characteristics and also they are free of leachable impurities [3].

10. In Biodegradable polymers, the system is biocompatible and it will not show dose leaving behind at any time and the polymer will keep its properties until after exhaustion of the drug.

11. In hydrogels like drug delivery systems, the properties of polymer materials like PEG,(the easy polymer used to design hydrogels), can be managed to enhance features like size of the pore, which is used to manage rate of diffusion of the conveyer drugs. PEGylation was considered to minister many diseases like hepatits B and C, neutropaenia connected with cancer chemotherapy (PEG-GCSF) 28 and various types of cancers [PEG] glutaminase merged with a glutamine anti-metabolite 6-diazo-5-oxo-norleucine (DON].

12. Polymers span from their use as films or binders covering agents in tablets to flow managing agent in liquids or emulsions for improving drug security and to alter the delivering characteristics. Micelles due to its smaller size have a small circulation time in the body. Hence, it results in an advantage of entering in the tumour cells easily, because of the EPR effect.

13. Large importance of polymers in drug delivery has been noticed because they give a distinctive property which so far is not achieved by any of the materials [4]. 
14. Polymers are preferable in the fact that they habitually show a pharmacokinetic profile as contrast to small-scale molecule drug with lengthy circulation time and they also have the ability for tissue targeting.

15. Gold nanoparticles are easy to prepare, good capability of co existence, and their capacity to attach with other biomolecules without changing their properties.

16. Biggest benefit of utilizing polymers in drug delivery is their control (manipulation) on their properties (e.g. linkers and molecular weight) to modify to the need of drug delivery systems.

\section{Difficulties and challenges}

1. Difficult to scale the process up and production in high amounts is expensive as microspheres are batch operations inherently [9].

2. It is possible to reproduce the distribution of size of the microsphere particles but the result is not uniform generally and the standard deviation that we get is equal to half of the average size. This is quite common. The distribution of the size should be as narrow as possible since the rate at which the drug will be released as well as syringability depends on the size of the sphere directly.

3. With the presence of organic solvents and aqueous-organic interfaces on drugs that are encapsulated leads to adverse effects like eliminating the bioactivity of microspheres.

4. It is not an easy task to remove the organic solvents totally as mostly they are toxic and there should be a regulation on the concentration of residual solvents in the microsphere.

5. A crucial limitation in the development of biodegradable polymer microspheres for controlled-release drug delivery applications is the difficulty of specifically designing systems that exhibit precisely controlled release rates.

6. Core-shell microparticles are significantly more difficult to manufacture than solid microspheres.

7. Handling and fabricating the microspehre's architecture is not easy as its shell and core must be immiscible.

8. Hydrogels have an ability to rapidly swell with water which may lead to faster release of the loaded drug than desired followed by the degradation of the polymer [10]. There is a period of release of hours to days for hydrophilic drugs that are delivered usinghydrogel systems and it is considered to be much lesser than hydrophobic polymers based delivery systems like microspheres or nanospheres.

9. There is a probability of controllable drug administration through the electrical stimulation of conducting polymers. One of the examples of such a polymer is polyprrole (Ppy) [11]. But they are not used generally as they have limitations related to the choice of dopant and molecular weight of the delivered drug.

10. A hindrance to oral administration of some classes of drugs, mainly peptides and proteins is caused due to hepatic first-pass metabolism [12] and degradation by enzymes within the gastrointestinal tract.

11. There are limitations of the mucosal surface for drug delivery as well. The first limitation being the low flux associated with mucosal delivery and the second as well as a major limitation of the trans mucosal route of administration is at the site of absorption due to lack of dosage form retention.

12. The conventional chemotherapeutic agents (using Nano scale polymers as carriers) that we are aware of work by destroying the cells that rapidly divide. This leads to the damage of normal healthy cells that divide rapidly such as cells in the macrophages, bone marrow, digestive tract, and hair follicles due to chemotherapy.

13. There are some side effects in many chemotherapeutic agents that includes mucositis (lining of the digestive tract affected by inflammation), loss of hair (alopecia), myelosuppression (white blood cells production is reduced leading to immunosuppression),dysfunction of the organ, and even anemia or thrombocytopenia [13]. These side effects lead to some difficulties like they impose dose reduction, treatment delay, or the given therapy is not continuous.

14. The cell division may be efficiently stopped near the center in solid tumor cells because of which chemotherapeutic agents become insensitive to chemotherapy.

15. Most of the times chemotherapeutic agents cannot penetrate and reach the core of solid tumors because of which they fail to kill the cancerous cells.

16. Most of the traditional chemotherapeutic agents often get excreted from the circulation being engulfed by macrophages and therefore they remain in the circulation for a very short time and cannot interact with the cancerous cells that lead to ineffectiveness of the chemotherapy.

17. Collagen has a limitation that it causes immunogenic responses in some patients therefore is not fit for use. It has a variant, atelocollagen. Atelocollagen preparation involves removal of the telopeptide from collagen. It has also been used for decrease in the potential immunogenicity. Collagen also has a poor mechanical strength 
and it cannot easily develop reproducible release rates [14].

18. Gelatin is cross-linked with glutaraldehyde while preparing the drug delivery system. This binds to and inactivates some protein drugs.

\section{Drug Delivery Devices-Requirements of Polymers in Drug Delivery System}

\subsection{Dendrimers}

Dendrimers are hyperbranched, monodisperse (uniform size particles in a dispersed phase), 3-D molecules of size 1 - $100 \mathrm{~nm}$ macromolecules. They solubilize by accommodating both hydrophobic and hydrophilic drugs. They consist of 3 structural components,

1. Central core (multifunctional)

2. Branched units

3. Surface groups

They are being used in the delivery of drugs and in other therapeutic agents at the specific sites. Drug can be encapsulated in the interior of the dendrimers (Figure 4) or can be adsorbed on and conjugated to the surface groups [2]. Sialylated dendrimers are inhibitors of the haemagglutinin of human erythrocytes by influenza viruses [2].

The dendrimers are water soluble and on hydrolysis releases free 5FU. Such dendrimers are very useful carriers for anti-tumour drugs [2]. Dendrimers also act as carriers (vectors), in gene therapy [26]. PANAM (polyamidoamine) dendrimers are also being used as carrier of genetic material. The amino groups at the dendrimer end react with phosphate groups of nucleic acids and form transfection complexes. For preserving the activity of DNA during dehydration, the transfection complexes are encapsulated in hydrophilic polymer, and then they are deposited on or sandwiched between fast degradation rate functional polymer films to mediate gene transfection [2].

\subsection{Polymeric-Nano Particulate Systems}

On the basis of method of preparation these can be Nanocapsules or Nanospheres.

\section{Microspheres and microcapsules}

Micro/Nano spheres are matrix system in which the drug is dispersed within the polymer throughout the body of particle. Micro/Nano capsules are vesicular systems in which cavity contains drug (oily/aqueous core) and is

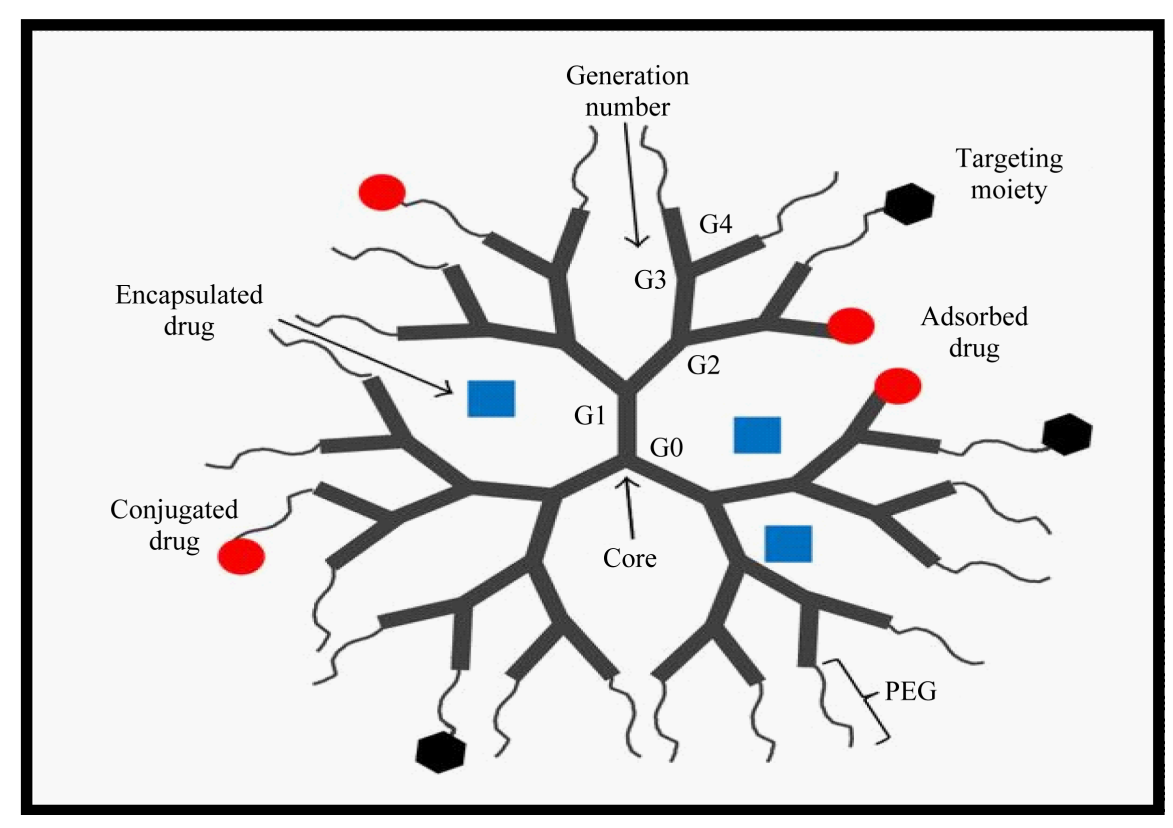

Figure 4. Simultaneous delivery of hydrophilic and hydrophobic drugs by encapsulation within hydrophobic cavities inside branching clefts, adsorption to the surface (ionic interaction) or direct covalent conjugation with functional groups on the surface [15]. 
surrounded by a single ultrathin membrane of polymer (reservoir systems for controlled release of drug) as depicted in Figure 5.

Drugs are released from the micro/nanosphere and micro/nanocapsule by diffusion through the polymer or by degradation of the polymer. Micro/nanospheres and micro/nanocapsules can be injected or taken orally. Lupron Depot is an injectable microsphere which is made up of lactic acid-glycolic acid copolymer and leuprolide acetate and entraps LHRH in order to treat prostate cancer [2].

\subsection{Hydrogel System}

Hydrogels are cross-linked networks of water-soluble polymers and are three dimensional. Hydrogels can be made both from natural and synthetic polymers. They are highly absorbent. Biodegradable hydrogels are being used as carriers for controlled drug delivery because of their inertness for many drugs and their biocompatibility. Hydrogels have very high porosity due to which the release rate of drug crucially depends upon the diffusion coefficient of the drug molecules. The tailoring of porosity of hydrogel can be done by controlling the level of cross-linking, which in turn affect the rate of delivery of the entrapped drug particles. The ability of hydrogels to rapidly swell in aqueous medium, promote the rate of release of the entrapped drug and degradation of the polymer [2]. Drug delivery through hydrogels is explained in Figure 6.

\subsection{Solid Lipid Nanoparticles}

Solid lipid nanoparticles are carrier system in which melted lipid is dispersed in an aqueous surfactant by micro

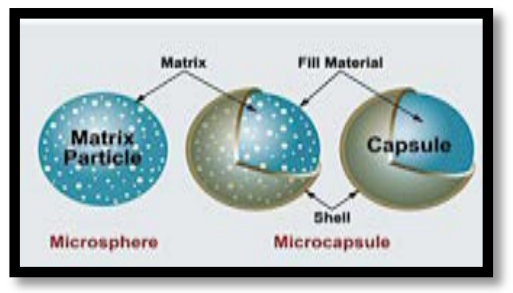

Figure 5. Microsphere and microcapsule [16].

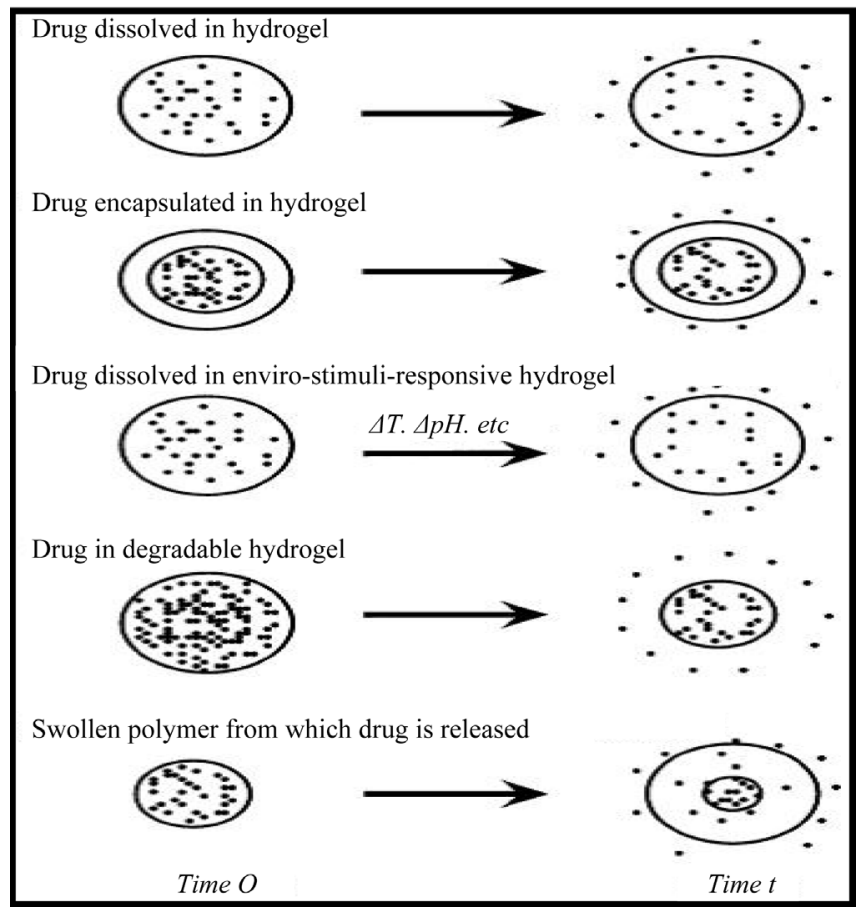

Figure 6. Hydrogel [17]. 
emulsification or high pressure homogenization. They are stable colloidal system with solid hydrophobic core. The core contains the dispersed or dissolved drugs. Surface coating with hydrophilic polymers like Polyethylene glycol (PEG) minimizes their uptake by liver and enhances bioavailability. It entraps both hydrophilic and lipophilic drugs. Solid lipids nanoparticles containing ibuprofen have been blended into dextran hydrogels are suitable for oral formulations.

\subsection{Magnetic Nanoparticles (MNPS)}

Drugs are bound with magnetic nanoparticles e.g. oxidized iron or magnetite (dextran coated) and are injected into blood stream. A high power magnetic field is generated outside the bodies which pull these drugs out of suspension and deliver the drug to a localized disease site. Coating these with dextran or PEG makes them stable water dispersible system.

\subsection{Polymeric Micelles (PMS)}

Polymeric micelles have a core shell structure formed by spontaneous self-assembly of individual amphiphilic di/tri block co-polymers (shown in Figure 7). They have both hydrophilic and hydrophobic regions which is good for drugs with poor solubility. Core is formed by hydrophobic block polymer blocks (poly-(propene glycol), poly-(caprolactone) etc.) and shell is made up of hydroplilic polymer (PEG).

\subsection{Liposomes}

Liposomes are vesicles composed of phospholipids and cholesterol. They are amphiphilic. The internal aqueous core is suited for delivery of hydrophilic drugs and the phospholipid bilayer encapsulates hydrophobic drugs. The modification of surface by attaching dextran or PEG to the phospholipid bilayer increases their circulation time in blood.

\subsection{Implants}

In most of the implants (a drug delivery system), a permeable polymeric membrane surrounds the core of solid drugs. The implants can be modified into different shapes, such as films, pellets, plugs, rods and discs [2]. The implants can be classified as non-biodegradable and biodegradable implants, depending on the polymer used. The polymers mostly used in the non-biodegradable implants include polyvinyl alcohol (PVA), silicone and ethylene vinyl acetate (EVA). Silicone can be customized to be both a permeable or impermeable layer depending on the grade and thickness of silicone used. Biodegradable systems can be made either by natural polymers (e.g. albumin, gelatin and collagen) or by synthetic polymers, such as Polylactic acid, olyglycolic acid and polylactic-co-glycolic acid (PLGA) copolymer [2]. In biodegradable implants, drug release occurs during polymer degradation. Implants approved by the FDA for eye: Retisert, Vitrasert, and Ozurdex [2]. Retisert, is an intraocular implant for the treatment of noninfectious uveitis that contains fluocinolone acetonide (FA). It is composed of an FA tablet containing PVA, magnesium stearate and microcrystalline cellulose. Vitrasert, is an intraocular implant that contains ganciclovir surrounded by PVA/EVA. It release drug in controlled fashion and is used to treat cytomegalovirus (CMV) retinitis. Ozurdex, is a biodegradable sustained release intravitreal implant that delivers dexamethasone to the vitreous humor and retina in order to treat macular edema and noninfectious posterior uveitis.

\section{Application Scopes of Polymers in Drug Delivey System}

Polymers are playing important role in pharmaceuticals. They are used as binders in tablet, increases solubility of poorly soluble drugs, used as film coatings on drugs to disguise their taste and enhances their stability etc. Some polymers which are used in drugs are discussed below.

\subsection{Biodegradable Polymers}

Biodegradable polymers have either hydrolytically or proteolytically labile bond in their backbone to make it chemically degradable [18]. At present two types of biodegradable polymers exists: natural polymers and synthetic polymers. Collagen and gelatin are two natural biodegradable polymers that are mostly used in drugs [19]. 


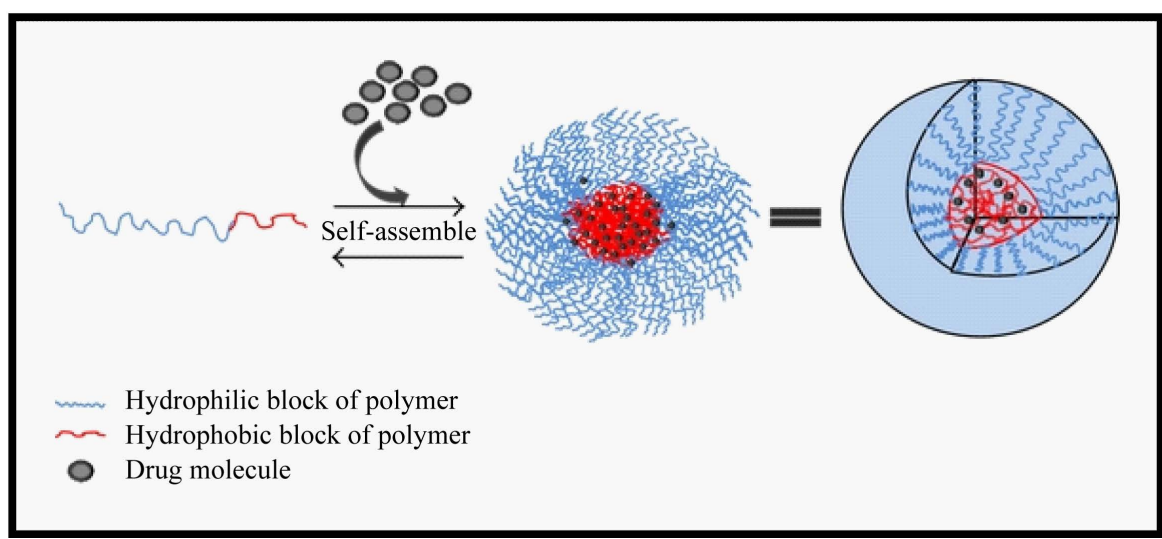

Figure 7. Drug loading of PMs by self-assembly of amphiphilic block copolymers in aqueous solution [25].

Collagens are biocompatible, non-toxic, can be easily isolated and purified in large quantities. Gelatin is a thermoreversible polymer [19]. Gelatin is easily available, have low antigen profile and have low binding affinity to drug molecules. All these properties make it suitable for drug delivery. Gelatin is cross-linked with glutaraldehyde to prepare it for drug delivery system. Synthetic biodegradable polymers are also present that include PLA, PLGA, PGA, poly(phosphazenes), poly(caprolactone), poly(anhydride), poly(phosphoesters), poly(cyanoacrylates), poly(acrylic acid), poly(amides), poly(ortho esters), polyethylene glycol, and polyvinyl alcohol and poly (isobutylcynoacrylate), poly(ethylene oxide), and poly(paradioxane). Among these, PLGA, the copolymer of PLA and PGA are mostly used polymers in drug delivery [18] [23]. Large numbers of biodegradable synthetic polymers rely on the hydrolytic cleavage of ester bonds [18].

- Polyethylene glycol: Polyethylene glycol is a hydrophilic polymer. Some features like low toxicity, lack of immunogenicity, antigenicity and excellent biocompatibility make it preffered polymer [18]. Its hydrophilic nature provides the protection to protein from any immune response.

- Polyesters: They have esters bond in the main chain [19]. Due to their biocompatible and biodegradable feature, PLA, PGA and their copolymer PLGA and poly (caprolactone) have been extensively used.

- Polyanhydrides: Polyanhydrides are biocompatible and bioabsorbable materials. They can be easily removed from the body because they can be degraded into their diacid counter parts in vivo [19].

- Polyamides: They contain the repeated unit of amide group and are hydrophilic in nature. Due to the presence of amide groups and hydrogen bonds, they have good mechanical properties and show high polar behaviour [19]. They are used to deliver low molecular weight drugs.

- Polyorthoesters: A number of studies have been done on the use of polyorthoesters as encapsulating material for various drugs.

- Polyaprolctone: PCL have been taken into consideration to be used as implantable biomaterial because it has ester linkage that can be hydrolysed in physiological conditions. It can also be used for preparation of long term implantable devices because it degrades very slowly.

\subsection{Non-Biodegradable Polymers}

Non-biodegradable polymers are commonly used in diffusion-controlled system [20]. Due to non biodegradable polymers, there is no initial burst release in diffusion-controlled systems. The permeability and thickness of the polymer, the solubility and the release area of the drug determines the release kinetics of the drug form the diffusion controlled system. Silicone, cross-linked Polyvinyl Alcohol, and Ethyl Vinyl Acetate are mostly used in drug formulations. Silicones are used as permeable or impermeable material. The permeability or the impermeability of the silicone material is decided by the thickness and the grade used. EVA is impermeable to many drugs, thus, commonly used as a membrane to surround the drug core. There is reduction in the release area due to EVA membrane, thus reduces the drug release rate. PVA is used as controlled elution membrane in the release area because they are permeable to various lipophilic drugs. Alteration in the thickness layer helps in achieving the desired release kinetics. 


\subsection{Smart Polymers}

They are high performance polymers which change according to the environment they are residing in. Even a small change in the environment can bring large changes in the polymer's properties. They can change the conformation, adhesiveness and water retention properties in response to $\mathrm{pH}$ change. They are used for production of hydrogels and other materials. These properties of smart polymers make them suitable for utilization in drug formulations. Some smart polymer are formed by the cross linking of the $\mathrm{pH}$ sensitive smart polymeric chains. The polymer composition, the nature of the ionizable groups, the hydrophilicity of the polymer backbone and the cross linking density decide the behaviour of the smart polymers. The cross linking density affects the permeability of the solute inversely, the higher the cross linking density, the lower the permeability [27]. Alginate gel beads are co-precipitated with a biologically active agent to form a sustained release gels. This gives the advantage of high loading of drugs while achieving better protein stability. LCST is a polymer, which have been tested in controlling drug delivery matrices. Copolymerisation of the NIPAAm with alkyl methacrylates maintains the temperature sensitivity because it increases its mechanical strength. There is reduction in the transportation of the bioactive molecules out of the polymers by surrounding the LCST with a thick layer of poly NIPAAm polymer [27].

\subsection{Gels}

These are hydrophilic polymers and have linear structures used in topical drug delivery. Linear structure is formed by covalent bonding between monomer units such as amides, ester, orthoesters, and glycosidic bonds [19]. Topical polymers are mostly prepared by organic polymers such as carbomers [21]. They are prepared by natural or synthetic polymers. Polymers which are used in its preparation include the natural gums tragacanth, pectin, agar, alginic acid and carrageenan; semi synthetic materials such as hydroxyethyl cellulose, methylcellulose, carboxymethyl cellulose and hydroxypropylmethyl cellulose; and the synthetic polymer, carbopol [21].

\subsection{Polymers in Mucoadhesive Delivery}

For developing the liquid ocular delivery system, the hydrophilic polymers should be used because they can be used as viscosity modifying or enhancing agent. Polysaccharides are frequently used in the ocular mucoadhesive delivery system. Its derivatives are hyaluronic acid, methyl cellulose, hydroxypropyl methylcellulose, gellan gum, chitosan, xanthan gum, carrageenan and guargum. Chitosan is a polysaccharide polymer. Its biodegradable, low toxic and biocompatible properties make it suitable for use in drug formulations [19]. Some other used nonionic polymers for mucoadhesive properties are poloxamer, polyvinylpyrrolidone and polyvinyl alcohol [22].

\subsection{Polymer Drug Conjugate Used for Cancer Treatment}

There is a physiological labile bond between the drug and the polymer. Paclitaxil [poly(L-glutamic acid)] is used as a chemotherapeutic agent to treat ovarian, breast and lung cancer. It has been studied in phase III trials. It has an ester linkage between its 2'hydroxyl group and the carboxylic acid of poly(L-glutamic acid) [19]. PEG and PAMAM are covalently conjugated with a chemotherapeutic drug Paclitaxil to increase its efficiency as an anticancer drug delivery system. Both increase its solubility. After an in-vitro study on human ovarian cancer cell it was found that PEG based conjugate reduced the activity of the paclitaxil by 25 -fold and the PAMAM-G4 dendrimer increases its efficiency by more than 10 times [18]. 5-flourouracil drug causes cell death. Nagarwal et al. Synthesized an encapsulating agent nanospheres of PLA polymer for 5-flurouracil [18].

\section{Conclusion}

Polymers are quite advantageous in drug delivery. This leads to enhanced drug delivery with better pharmacokinetics handling all safety parameters. Mechanism and time taken for drug delivery system for a particular tissue or cellular compartment still needs to be studied. In order to design the most suitable polymer therapeutic many queries including gene delivery p need to be answered as well. This leads to the synthesis of the smart polymer. In targeted drug delivery systems the site of action should be clearly known. Biocompatible polymers provide better control over the toxicity of the samples; this leads to more reliable drug delivery and ensures patient's safety. Novel strategies like dendrimer synthesis and controlled polymerisation techniques are now well 
established. Development of therapeutics at nano level with novel improved features utilizing polymeric materials to address unresolved medical needs and to enable the application of personalized medicine is still required. Currently, the main goals in this field are to enable and support further research efforts towards translatable and competitive product development. With this as a goal, the rapid and successful translation of emerging polymeric drug delivery systems in the future can be derived by interdisciplinary and open-minded approach.

\section{References}

[1] Schmaljohann, D. (2006) Thermo and pH Responsive Polymers in Drug Delivery. Advanced Drug Delivery Reviews, 58, 1655-1670. http://dx.doi.org/10.1016/j.addr.2006.09.020

[2] Liechty, W.B., et al. (2010) Polymers for Drug Delivery Systems. Annual Review of Chemical and Biomolecular Engineering, 1, 149-173. http://dx.doi.org/10.1146/annurev-chembioeng-073009-100847

[3] Pallerlaand, S. and Prabhakar, B. (2013) Review on Polymers in Drug Delivery. American Journal of Pharmtech Research, 3, 901-917.

[4] Biomaterials Tutorial. http://www.sigmaaldrich.com/material science/biomaterials/tutorial.html

[5] Jawahar, N. and Meyyanathan, S.N. (2012) Polymeric Nanoparticles for Drug Delivery and Targeting: A Comprehensive Review. International Journal of Health and Allied Sciences, 1, 217-223. http://dx.doi.org/10.4103/2278-344X.107832

[6] Shaik, M.R., Korsapati, M. and Panati, D. (2012) Polymers in Controlled Drug Delivery Systems. International Journal of Pharma Sciences, 2, 112-116.

[7] Yang, W. and Pierstorff, E. (2012) Reservoir Based Polymer Drug Delivery Systems. Journal of Laboratory Automation, 17, 50-58. http://dx.doi.org/10.1177/2211068211428189

[8] Srikanth, P., Raju, N., Raja, S.W. and Raj, S.B. (2013) A Review on Oral Controlled Drug Delivery. International Journal of Advanced Pharmaceutics, 3, 51-58.

[9] Kim, K.K. and Pack, D.W. (2006) Microspheres for Drug Delivery. 19-50.

[10] Hoare, T.R. and Kohane, D.S. (2008) Hydrogels in Drug Delivery: Progress and Challenges. Polymer, 49, $1993-2007$. http://dx.doi.org/10.1016/j.polymer.2008.01.027

[11] George, P.M., LaVan, D.A., Burdick, J.A., Chen, C.-Y., Liang, E. and Langer, R. (2006) Electrically Controlled Drug Delivery from Biotin-Doped Conductive Polypyrrole. Advanced Materials, 18, 577-581. http://dx.doi.org/10.1002/adma.200501242

[12] Patel, V.F., Liu, F. and Brown, M.B. (2011) Advances in Oral Transmucosal Drug Delivery. Journal of Controlled Release, 153, 106-116. http://dx.doi.org/10.1016/j.jconrel.2011.01.027

[13] Sutradhar, K.B. and Amin, M.L. (2014) Nanotechnology in Cancer Drug Delivery and Selective Targeting. ISRN Nanotechnology, 2014, Article ID: 939378.

[14] Park, J.-H., Allen, M.G. and Prausnitz, M.R. (2005) Biodegradable Polymers Microneedles: Fabrication, Mechanics and Transdermal Drug Delivery. Journal of Controlled Release, 104, 51-66. http://dx.doi.org/10.1016/j.jconrel.2005.02.002

[15] Lee, J.H. and Nan, A. (2012) Combination Drug Delivery Approaches in Metastatic Breast Cancer. Journal of Drug Delivery, 2012, Article ID: 915375.

[16] Shanmugam, S., Reddy, J.S. and Vetrichelvan, T. Formulation and in Vitro Evaluation of 5-Fluorouracil Microcapsules by Using Different Methods of Micro Encapsulation. http://www.pharmatutor.org/articles/formulation-invitro-evaluation-5-fluorouracil-microcapsules-different-methods-mi croencapsulation

[17] Peter, G., Emmanuelle, R. and Daniel, S. (2012) Hydrophilic Polymer Networks with Environmental Sensitivity (New Nani Formulations). http://www.flintbox.com/public/project/19743

[18] Liechty, W.B., Kryscio, D.R., Slaughter, B.V. and Peppas, N.A. (2010) Polymers for Drug Delivery Systems. Annual Review of Chemical and Biomolecular Engineering, 1, 149-173. http://dx.doi.org/10.1146/annurev-chembioeng-073009-100847

[19] Vilar, G., Tulla-Puche, J. and Albericio, F. (2012) Polymers and Drug Delivery Systems. Current Drug Delivery, 9, 367-394.

[20] Yang, W.-W. and Pierstorff, E. (2012) Reservoir-Based Polymer Drug Delivery Systems. Journal of Laboratory Automation, 17, 50-58.

[21] Bhowmik, D., Gopinath, H., Kumar, B.P., Duraivel, S. and Sampath Kumar, K.P. (2012) Recent Advances in Novel Topical Drug Delivery System. The Pharma Innovation, 1, 12-31. 
[22] Yousef, J. and Sanaz, H. (2012) Novel Drug Delivery Systems for Modulation of Gastrointestinal Transit Time. In: Sezer, A.D., Ed., Recent Advances in Novel Drug Carrier Systems, InTech Publisher, Rijeka.

[23] Martinho, N., Damgé, C. and Reis, C.P. (2011) Recent Advances in Drug Delivery Systems. Journal of Biomaterials and Nanobiotechnology, 2, 510-526. http://dx.doi.org/10.4236/jbnb.2011.225062

[24] Ahmed, E.M. (2013) Hydrogel: Preparation, Characterization, and Applications: A Review. Journal of Advanced Research, 6, 105-121. http://dx.doi.org/10.1016/j.jare.2013.07.006

[25] Xu, W., Ling, P.X., and Zhang, T.M. (2013) Polymeric Micelles, a Promising Drug Delivery System to Enhance Bioavailability of Poorly Water-Soluble Drugs. Journal of Drug Delivery, 2013, Article ID: 340315.

[26] Madaan, K., Kumar, S., Poonia, N., Lather, V. and Pandita, D. (2014) Dendrimers in Drug Delivery and Targeting: Drug-Dendrimer Interactions and Toxicity Issues. Journal of Pharmacy and Bioallied Sciences, 6, 139-150. http://dx.doi.org/10.4103/0975-7406.130965

[27] Mahajan, A. and Aggarwal, G. (2011) Smart Polymers: Innovations in Novel Drug Delivery. International Journal of Drug Development \& Research, 3, 16-30. 


\section{Abbreviations}

P(MAA-g-EG): Poly(methacrylic acid-grafted-poly(ethylene glycol))

PNIPAAm: Poly(N-isopropylacrylamide)

PVA: Polyvinyl alcohol

PLGA: poly(lactic-co-glycolic acid)

PAMAM: Poly(amidoamine)

PGA: Polyglycolic acid

PLA: Polylactic acid

DNA: Deoxyribonucleic acid

FDA: Food and Drug Administration

LCST: lower critical solution temperature

EVA: Ethylene-vinyl acetate

PEG: Polyethylene glycol

DON: Diazo-5-oxonorleucine

Ppy: Polypyrrole

FA: Fluocinolone acetonide

CMV: Cytomegalo virus 